\title{
Improved Stiffness Modeling for An Exechon-Like Parallel Kinematic Machine (PKM) and Its Application
}

Nanyan Shen ${ }^{1,2}$, Liang Geng ${ }^{1,2}$, Jing $\mathrm{Li}^{1,2^{*}}$, Fei Ye ${ }^{1,2}$, Zhuang $\mathrm{Yu}^{1,2}$ and Zirui Wang ${ }^{1,2}$

\begin{abstract}
Hole drilling or contour milling for the large and complex workpieces such as automobile panels and aircraft fuselages makes a high combined demand on machining accuracy, stiffness and workspace of machining equipment. Therefore, a 5-DOF (degrees of freedom) parallel kinematic machine (PKM) with redundant constraints is proposed. Based on the kinematics analysis of the parallel mechanism using intermediate variables, the kinematics problems of the PKM are solved through equivalent kinematics model. The structural stiffness matrix method is adopted to model the stiffness of the parallel mechanism of the PKM, where the stiffness of each joint and branch component is obtained by stiffness formula and finite element analysis. And the stiffness model of the parallel mechanism is improved by correction coefficient matrix, each element of which is constructed as a polynomial function of three independent end variables of the parallel mechanism. The terminal stiffness matrices obtained by simulation result are used to determine the coefficients of polynomial function by least square fitting to describe the correction coefficient over the workspace of the parallel mechanism quantitatively. The experiment results prove that the modification method can greatly improve the stiffness model of the parallel mechanism. To enhance the machining accuracy of the PKM, the proposed kinematics model and the improved stiffness model are utilized to optimize the working stiffness of parallel machine by searching the best relative position of parallel machine and workpiece. A plate workpiece taken as example is examined in the case study section, which demonstrates the effectiveness of optimization method.
\end{abstract}

Keywords: PKM, 5-DOF, Equivalent kinematics model, Intermediate variables, Stiffness correction coefficient, Optimal working stiffness

\section{Introduction}

The parallel kinematic machine (PKM) is composed of parallel mechanism and series mechanism, which have both the characteristics of these two types of mechanism. Therefore, compared with the conventional industrial robot, the PKM has more advantages in material removal applications, which require higher stiffness and precision of machining equipment in a relatively large workspace than other applications, such as feeding, welding and printing.

\footnotetext{
*Correspondence: ian1982@shu.edu.cn

1 Shanghai Key Laboratory of Intelligent Manufacturing and Robotics,

Shanghai University, Shanghai 200444, China

Full list of author information is available at the end of the article
}

Recently, there are increasing practical applications of PKMs in the industries of automobile and aviation [1-3].

Many PKMs with different structures have been proposed, among which the most representative ones are Tricept mechanism and Exechon mechanism $[4,5]$. Bi et al. $[6,7]$ proposed an Exechon parallel mechanism with redundant constraints, which had three DOF, and presented a method of solving the kinematics problem for Exechon parallel mechanism by using intermediate variables to establish the relation between joint variables and end variables. Zhang et al. [8, 9] proposed a Tricept parallel mechanism, the stiffness of which was enhanced by a passive chain, and used various intelligent algorithms to evaluate the positive kinematics solution of a 3-DOF 
parallel mechanism with redundant actuation and found that $\mathrm{v}-\mathrm{SVM}$ was the most efficient algorithm.

As an effective way to evaluate the performance of PKM, stiffness analysis is usually separated into the analysis on terminal stiffness of parallel mechanism and joint stiffness of series mechanism [10-13]. Bi et al. [14] used finite element method and structural stiffness matrix to solve the terminal stiffness of Exechon parallel mechanism. Dumas [15] introduced a general method for calculating the stiffness of any 6-DOF series robot by fining out its joint stiffness.

Based on the stiffness analysis, the stiffness model can be transferred into computer algorithms for applications on mechanical design and machining. Fan et al. [16] related to the design of PKM for optimal static stiffness using computer simulation. He et al. [17] proposed a new stiffness orientation method, which was used to optimize milling path and improve stability of the robotic milling system. Li et al. [18] used robot stiffness model and dynamic milling force model to optimize the cutter path and clamping position in robotic milling process.

In this paper, a new type of redundant constrained PKM is proposed, and its kinematics and stiffness are studied in detail. Section 2 analyzes the structure of the PKM. Section 3 introduces its kinematics solution. The improved stiffness model of the parallel mechanism is established in Section 4. The following section shows the effectiveness of the improved stiffness model and analysis on the stiffness of the parallel mechanism. Section 6 develops an application of the kinematics model and the improved stiffness model to improve the machining accuracy of the PKM. In Section 7, some findings of this research are concluded.

\section{Structure of PKM}

As shown in Figure 1, the PKM with redundant constraints is composed of a base, a fixed platform, three limbs, a moving platform, two rotary joints and a spindle. Limb1 and limb2 are two symmetrical linear motion chains, which are connected with the fixed platform at one end via a Hooke hinge, and with the moving platform at the other end via a rotary pair. The third linear motion chain limb3 is connected to the fixed platform in the form of a rotary pair and to the moving platform via a spherical pair. The two rotary joints are mounted in series on the moving platform of the parallel mechanism, the rotary axes of which are perpendicular to each other. The spindle is mounted on the end of the PKM via a flange, which serves as the end-effector to drive the milling cutter.

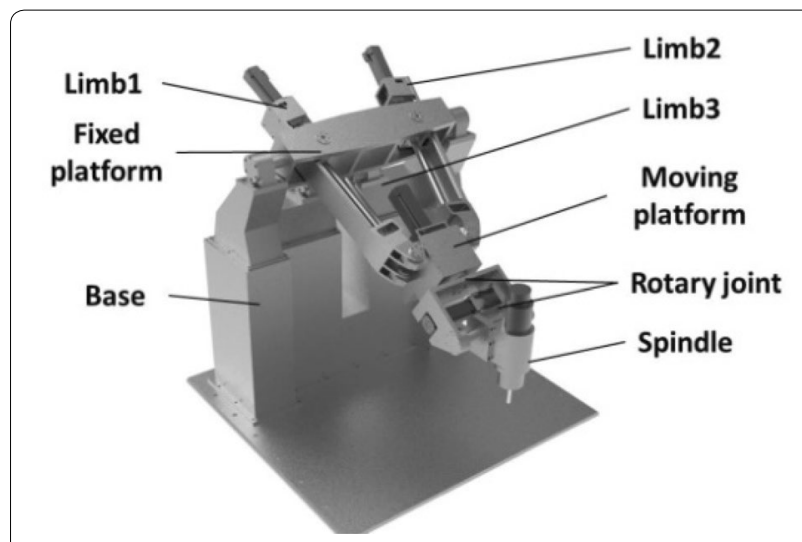

Figure 1 Structure of the PKM

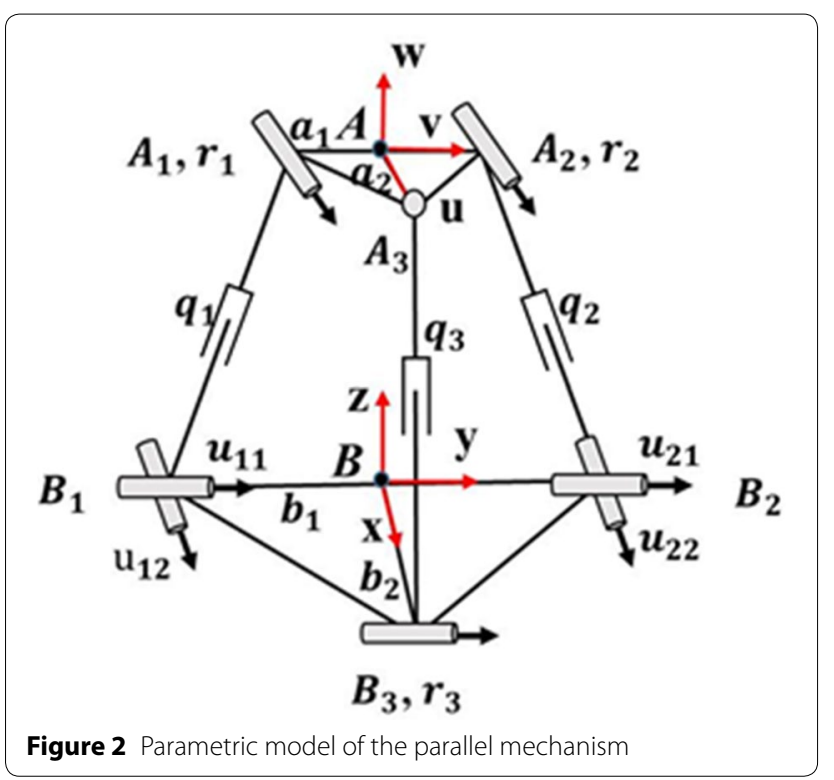

As an important feature of this new PKM, the parametric model of the parallel mechanism is shown in Figure 2, where $B_{1}, B_{2}$ and $B_{3}$ are the junctions of limb1, limb2, limb3 and fixed platform, respectively; $A_{1}, A_{2}$ and $A_{3}$ are the junctions of limb1, limb2, limb3 and moving platform, respectively. The joint variables $q_{i}$ $(i=1, \ldots, 3)$ of three branches represent the lengths of lines $A_{1} B_{1}, A_{2} B_{2}$ and $A_{3} B_{3} . \triangle A_{1} A_{2} A_{3}$ and $\triangle B_{1} B_{2} B_{3}$ are isosceles triangles, where sides $B_{1} B_{3}$ and $A_{1} A_{3}$ are equal to sides $B_{2} B_{3}$ and $A_{2} A_{3}$, respectively. Points $A$ and $B$ are the midpoints of sides $A_{1} A_{2}$ and $B_{1} B_{2}$. The lengths of sides $B B_{2}, B B_{3}, A A_{2}$ and $A A_{3}$ are $b_{1}, b_{2}, a_{1}$ and $a_{2}$, respectively. Coordinate systems $B-x y z$ and $A-u v w$ are established on the fixed platform and the moving platform, respectively. For coordinate system $B-x y z$, the direction of $x$ axis points from point $B$ to point $B_{3}$ and 
the direction of $y$ axis points from point $B$ to point $B_{2}$. For coordinate system $A-u v w$, the direction of $u$ axis points from point $A$ to point $A_{3}$ and the direction of $v$ axis points from point $A$ to point $A_{2}$. Besides the vectors $\boldsymbol{u}_{11}$ and $\boldsymbol{u}_{21}$ coincide with the rotary axes of the first joint of Hooke hinges of limb1 and limb2, respectively; the vectors $\boldsymbol{u}_{12}$ and $\boldsymbol{u}_{22}$ coincide with the rotary axes of the second joint of Hooke hinges of limb1 and limb2, respectively; the vectors $\boldsymbol{r}_{1}, \boldsymbol{r}_{2}$ and $\boldsymbol{r}_{3}$ coincide with the rotary axes of rotary joints of limb1, limb2 and limb3, respectively.

The Kutzbach Grubler formula are commonly used to calculate the degrees of freedom (DOF) of the mechanism and it is expressed as

$$
M=6(n-g-1)+\sum_{1}^{g} f_{i},
$$

where $M$ is the number of DOF of the mechanism; $n$ is the number of the rigid bodies in the mechanism, $g$ is the number of joints; and $f_{i}$ is the number of DOF for joint $i$.

Without consideration of the redundancy of constraints in the proposed the parallel mechanism, its DOF is equal to 1 according to Eq. (1), which should be adjusted to 3 by analyzing the extra DOF caused by redundant constraints [6, 7]. And then DOF of the whole PKM amounts to 5 , counting 2 DOF of two rotary joints mounted on the end of the parallel mechanism.

There are only 6 passive pairs in the proposed PKM less than those in Tricept mechanism, which is beneficial to the decrease in motion error caused by passive pairs clearance and the practical application of PKM [19]. The kinematic pairs in Exechon mechanism which connect limb3 to the fixed platform and to the moving platform are interchanged in the proposed PKM, which makes it possible to use one-piece precision spherical pair instead of three rotation pairs and thus avoid the assembly error.

\section{Kinematics Modeling}

In this paper, the kinematics analysis of the PKM is carried out by solving the equivalent kinematics model. Here, the parallel mechanism is regarded as a joint of the PKM and then it is equivalent to a 3-joint series mechanism. The first step of kinematics analysis is to establish $\mathrm{DH}$ model for the 3-joint series mechanism equivalent to the PKM. And then the kinematics analysis of the parallel mechanism of the PKM is discussed in detail to obtain the kinematics model of the PKM.

\subsection{Kinematics Model of the PKM}

The position and pose matrix $\boldsymbol{T}_{\mathrm{h}}$ and $\boldsymbol{T}_{\mathrm{p}}$ of the PKM and the parallel mechanism in coordinate system $B-x y z$ are solved by $x y z$ and $z y x$ Euler angles respectively.
The position and pose variation matrices $\boldsymbol{T}_{\mathrm{s} 1 i}$ and $\boldsymbol{T}_{\mathrm{s} 2 i}$ $(i=1,2)$ of two series joints can be obtained by establishing standard DH model according to the parameters listed in Table 1 , where $\beta$ and $\alpha$ are the amounts of rotation around $z$ and $x$ axes, respectively; $d$ and $b$ are the amounts of movement along $z$ and $x$ axes, respectively [20].

And then position and pose matrix $\boldsymbol{T}_{\mathrm{h}}$ of the PKM can be calculated by Eq. (2) according to the principle of coordinate transformation:

$$
\boldsymbol{T}_{\mathrm{h}}=\boldsymbol{T}_{\mathrm{p}} \boldsymbol{T}_{\mathrm{s} 11} \boldsymbol{T}_{\mathrm{s} 12} \boldsymbol{T}_{\mathrm{s} 21} \boldsymbol{T}_{\mathrm{s} 22} .
$$

By multiplying the inverse matrix of $\boldsymbol{T}_{\mathrm{s} 22}$ and $\boldsymbol{T}_{\mathrm{s} 21}$ on both sides of Eq. (2), the joint variables of the series mechanism and the end variables of the parallel mechanism can be obtained by letting the corresponding elements of matrix on both sides of the equation equal to each other. According to the end variables of the parallel mechanism, the inverse kinematics of the parallel mechanism is analyzed. And thus, the inverse kinematics solution of the PKM is achieved.

\subsection{Kinematics Model of the Parallel Mechanism}

To solve the kinematics model of the PKM, the kinematics model of the parallel mechanism is solved by the method of intermediate variables to determine its position and pose matrix $\boldsymbol{T}_{\mathrm{p}}$.

Through the analysis on the structure of the parallel mechanism, it can be known that the direction of $u$ axis of coordinate system $A-u v w$ is perpendicular to the directions of $y$ axis of coordinate system $B-x y z$ and vector $\boldsymbol{A}_{1} \boldsymbol{B}_{1}$; the direction of $y$ axis of coordinate system $B-x y z$ is perpendicular to the direction of vector $\boldsymbol{A}_{3} \boldsymbol{B}_{3}$. According to these constraint conditions, Eq. (3) is shown as below, which indicates that the workspace of the parallel mechanism is a plane:

$$
\left\{\begin{array}{l}
\varphi=0, \\
Y_{0}=0, \\
X_{0}=Z_{0} \times \tan \theta .
\end{array}\right.
$$

As shown in Figure 3, three intermediate variables are designed here through the analysis of the parallel mechanism, which include distance $L$ between points $A$ and $B$,

Table 1 Parameters of DH model of two series joints

\begin{tabular}{lllll}
\hline & $\boldsymbol{\beta}\left(^{\circ}\right)$ & $\boldsymbol{d}(\mathbf{m m})$ & $\boldsymbol{a}\left(^{\circ}\right)$ & $\boldsymbol{b}(\mathbf{m m})$ \\
\hline 1 & 0 & 0 & 0 & -20 \\
2 & $\beta_{1}$ & 417 & 90 & -35 \\
3 & $\beta_{2}$ & 0 & -90 & -150 \\
4 & 0 & 190 & 0 & 0 \\
\hline
\end{tabular}




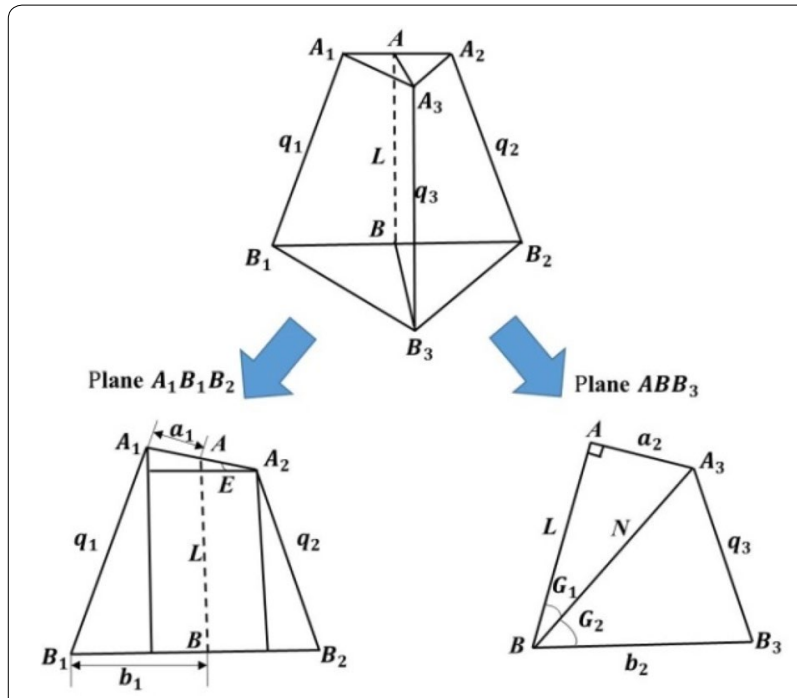

Figure 3 Schematic diagram of the parallel mechanism

angle $E$ between lines $A_{1} A_{2}$ and $B_{1} B_{2}$, angle $G$ between planes $A_{1} B_{1} B_{2}$ and $B_{1} B_{2} B_{3}$. Thus, the kinematics problem of the parallel mechanism can be solved by relating end variables to intermediate variables and also intermediate variables to joint variables, respectively.

Eq. (4) can be derived according to the geometric relations shown in Figure 3, where line $A B$ is perpendicular to lines $B_{1} B_{2}$ and $A A_{3}$. And the values of $L$ and $E$ can be obtained by the equation.

$$
\left\{\begin{array}{l}
\left(L+a_{1} \times \sin E\right)^{2}+\left(b_{1}-a_{1} \times \cos E\right)^{2}=q_{1}^{2} \\
\left(L-a_{1} \times \sin E\right)^{2}+\left(b_{1}-a_{1} \times \cos E\right)^{2}=q_{2}^{2}
\end{array}\right.
$$

Eq. (5) can be obtained from the geometric relations shown in Figure 3:

$$
\left\{\begin{array}{l}
G_{1}=a \tan \left(a_{2} / L\right) \\
N=\sqrt{L^{2}+a_{2}^{2}} \\
G_{2}=a \cos \left(\left(N^{2}+b_{2}^{2}-q_{3}^{2}\right) /\left(2 \times N \times b_{2}\right)\right), \\
G=G_{1}+G_{2}
\end{array}\right.
$$

Based on the above analysis, the values of intermediate variables $L, E, G$ can be obtained according to the values of joint variables $q_{1}, q_{2}$ and $q_{3}$. And then the values of end variables $\theta, \sigma$, and $Z_{0}$ can be evaluated according to the values of $L, E$ and $G$ via Eq. (6). Thus, the kinematics problem of the parallel mechanism is solved.

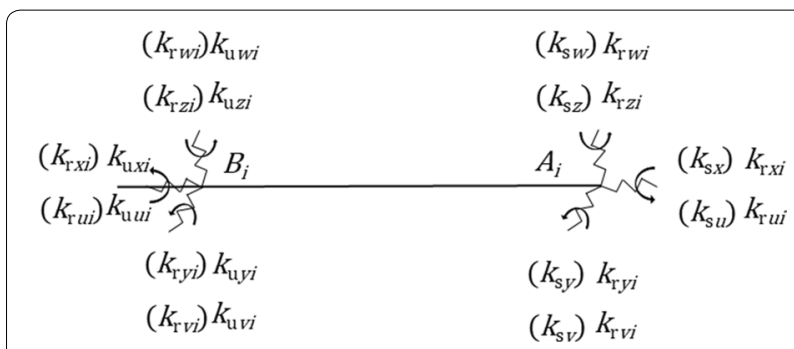

Figure 4 Simplified diagram of branch structure

$$
\left\{\begin{array}{l}
\theta=\frac{\pi}{2}-G \\
\sigma=-E \\
Z_{0}=L \times \sin G
\end{array}\right.
$$

\section{Kinetostatic Modeling}

The stiffness modeling of PKM includes the stiffness modeling of parallel mechanism and series mechanism. The paper focus on the stiffness modeling of parallel mechanism, because the stiffness of series mechanism has little impact on the stiffness of PKM [14]. And PKM has different forms of series mechanism for different applications, it is not meaningful to study the stiffness modeling of series mechanism [21]. As for the stiffness modeling of parallel mechanism, the stiffness modeling method for Exechon parallel mechanism is adopted to achieve the basic stiffness model [5, 21, 22]. In order to improve the accuracy of the basic stiffness model, correction coefficient matrix of the basic stiffness model is solved by regression analysis method to optimize the stiffness model and get the improved stiffness model.

\subsection{Basic Stiffness Model of Parallel Mechanism}

The terminal stiffness matrix of the parallel mechanism is affected by its three branches, each of which includes three parts. Limb1 and limb2 include Hooke hinge U, prismatic joint $\mathrm{P}$ and rotary joint $\mathrm{R}$. Limb3 includes rotary joint $R$, prismatic joint $P$ and spherical joint $S$. Therefore, it can be determined by analyzing the stiffness of three branches. As shown in Figure 4, the connecting joints in the parallel mechanism are replaced by the springs with equivalent stiffness. And the prismatic joint is regarded as a cantilever beam which is composed by a lead screw connected in parallel with a connecting rod. The fixed platform and the moving platform are regarded as rigid bodies. Thus, the terminal stiffness matrix of the parallel mechanism $\boldsymbol{K}_{\mathrm{p}}$ can be obtained without considering the influence of frictional force and damping force between the connecting members. 
To solve the stiffness of the parallel mechanism, the stiffness of each joint is solved firstly by finite element analysis and structural stiffness analysis [21], which can be expressed as Eq. (7):

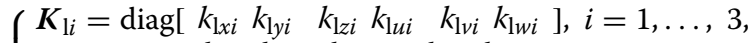

$$
\begin{aligned}
& \left\{\begin{array}{l}
\boldsymbol{K}_{\mathrm{r} i}=\operatorname{diag}\left[\begin{array}{lllll}
k_{\mathrm{r} x i} & k_{\mathrm{r} y i} & k_{\mathrm{r} z i} & 0 & k_{\mathrm{r} v i} \\
k_{\mathrm{r} w i}
\end{array}\right], i=1, \ldots, 3,
\end{array}\right. \\
& \left\{\boldsymbol{K}_{\mathrm{u} i}=\operatorname{diag}\left[\begin{array}{llllll}
k_{\mathrm{u} x i} & k_{\mathrm{u} y i} & k_{\mathrm{u} z i} & 0 & 0 & k_{\mathrm{u} w i}
\end{array}\right], i=1,2,\right. \\
& \boldsymbol{K}_{\mathrm{s}}=\operatorname{diag}\left[\begin{array}{lllllll}
k_{\mathrm{s} x} & k_{\mathrm{s} y} & k_{\mathrm{s} z} & 0 & 0 & 0
\end{array}\right],
\end{aligned}
$$

where subscript 1 represents the link of each branch; subscript $\mathrm{r}$ represents the rotary joint $R$; subscript $\mathrm{u}$ represents the Hooke hinge $U$; subscript $\mathrm{s}$ represents the spherical joint $S$; subscript $i$ represents the number of each branch. And the values of $k_{\mathrm{r} u i}, k_{\mathrm{u} u i v}, k_{\mathrm{u} v i}, k_{\mathrm{s} u}, k_{\mathrm{s} v}$ and $k_{\mathrm{s} w}$ are zero.

Based on the above analysis of joint stiffness of each branch, the terminal stiffness matrix of the parallel mechanism can be obtained by the structural stiffness matrix. The parallel mechanism can be divided into four units, the nodes of which are points $A_{1}, A_{2}, A_{3}$ and $A$. Hence, the structural stiffness matrix $K$ of the parallel mechanism can be expressed as Eq. (8):

$$
\boldsymbol{K}=\left[\begin{array}{cccc}
\boldsymbol{K}_{11} & 0 & 0 & \boldsymbol{K}_{14} \\
0 & \boldsymbol{K}_{22} & 0 & \boldsymbol{K}_{24} \\
0 & 0 & \boldsymbol{K}_{33} & \boldsymbol{K}_{34} \\
\boldsymbol{K}_{41} & \boldsymbol{K}_{42} & \boldsymbol{K}_{43} & \boldsymbol{K}_{44}
\end{array}\right]
$$

where $\boldsymbol{K}_{i i}(i=1, \ldots, 4)$ is the stiffness of four nodes; $\boldsymbol{K}_{4 i}(i=1, \ldots, 3)$ is the coupling stiffness relating the first three nodes to the fourth node; $\boldsymbol{K}_{i 4}(i=1, \ldots, 3)$ is the coupling stiffness relating the fourth node to the first three nodes.

The elements $\boldsymbol{K}_{i i}(i=1, \ldots, 4)$ in structural stiffness matrix $K$ are acquired through the analysis on force relation and displacement relation between the first three nodes $A_{1}, A_{2}, A_{3}$ and the fourth node $A$, and the deformation compatibility conditions of the parallel mechanism. The specific steps for solving $\boldsymbol{K}_{i i}$ is not listed here, which can be found by the stiffness modeling of Exechon PKM $[5,21,22]$.

It can be found that structural stiffness matrix $K$ is non-diagonal, which indicates that the terminal stiffness of the parallel mechanism is effected by the stiffnesses of three branches. The terminal stiffness matrix $\boldsymbol{K}_{\mathrm{p}}$ of the parallel mechanism in coordinate system $A-u \nu w$ can be determined by flexibility matrix [21]:

$$
\boldsymbol{K}_{\mathrm{p}}=\boldsymbol{T}_{\mathrm{p} 0}^{\prime}\left[\boldsymbol{K}_{6 \times 6}^{-1}\right]^{-1} \boldsymbol{T}_{\mathrm{p} 0}
$$

where $\boldsymbol{T}_{\mathrm{p} 0}=\operatorname{diag}\left[\boldsymbol{R}_{\mathrm{p} 0}, \boldsymbol{R}_{\mathrm{p} 0}\right], \boldsymbol{R}_{\mathrm{p} 0}$ represents the first $3 \times 3$ block of matrix $\boldsymbol{T}_{\mathrm{p}}, \boldsymbol{K}_{6 \times 6}^{-1}$ represents the last $6 \times 6$ block of the inverse matrix of $\boldsymbol{K}$.

\subsection{Improved Stiffness Model of the Parallel Mechanism}

In order to improve the stiffness model of the parallel mechanism, the correction coefficient matrix is introduced, each element of which is a function of three independent end variables of the parallel mechanism. Based on the stiffness data of simulation result, the polynomial function is constructed to fit the correction coefficient of stiffness calculated by stiffness model, which establishes the basis of the improved stiffness model. The construction method is presented in detail and verified by its good effect on improving the precision of stiffness model.

Because the diagonal elements in the stiffness matrix are the principle stiffness values, the non-diagonal elements are the coupled stiffness values, so we only analyze the principle stiffness values for content limitation [21]. The correction coefficient matrix $C_{\mathrm{p}}$ is defined as a diagonal matrix shown in Eq. (10) accordingly. Then the improved stiffness matrix $\boldsymbol{K}_{\mathrm{m}}$ can be acquired by calculating the dot product of correction coefficient matrix $C_{\mathrm{p}}$ and stiffness matrix $\boldsymbol{K}_{\mathrm{p}}$ obtained by stiffness model.

$$
\left\{\begin{array}{l}
\boldsymbol{K}_{\mathrm{m}}=\boldsymbol{C}_{\mathrm{p}} \times \boldsymbol{K}_{\mathrm{p}} \\
\boldsymbol{C}_{\mathrm{p}}=\operatorname{diag}\left[c_{\mathrm{p} 11}, c_{\mathrm{p} 22}, c_{\mathrm{p} 33}, c_{\mathrm{p} 44}, c_{\mathrm{p} 55}, c_{\mathrm{p} 66}\right] .
\end{array}\right.
$$

Each diagonal element $c_{\mathrm{p} i i}(i=1, \ldots, 6)$ of correction coefficient matrix $C_{\mathrm{p}}$ can be expressed by a polynomial function of three independent end variables $\theta, \sigma$ and $Z_{0}$ of the parallel mechanism.

The terminal stiffness matrix $\boldsymbol{K}_{\mathrm{a}}$ of the parallel mechanism obtained by stiffness measurement experiment [23] or finite element analysis [24] is regarded as the improvement goal of stiffness model. In order to acquire the stiffness matrices of the parallel mechanism at a series of position and poses, an orthogonal experiment is conducted. The orthogonal experiment is designed by using the first three columns of $L_{49}\left(7^{8}\right)$ orthogonal experiment table to divide three variables $\theta, \sigma$ and $Z_{0}$ into seven levels, which can reduce the complexity of the experiment and also characterize the workspace of the parallel

Table 2 Levels of three independent end variables

\begin{tabular}{llllrrrr}
\hline & $\mathbf{1}$ & $\mathbf{2}$ & $\mathbf{3}$ & $\mathbf{4}$ & $\mathbf{5}$ & $\mathbf{6}$ & $\mathbf{7}$ \\
\hline$\theta\left(^{\circ}\right)$ & -15 & -10 & -5 & 0 & 5 & 10 & 15 \\
$\sigma\left(^{\circ}\right)$ & -15 & -10 & -5 & 0 & 5 & 10 & 15 \\
$Z_{0}(\mathrm{~mm})$ & 500 & 510 & 520 & 530 & 540 & 550 & 560 \\
\hline
\end{tabular}


mechanism. To cover the workspace as much as possible, the levels of each variable can be distributed as Table 2.

According to the orthogonal experiment, the terminal stiffness matrix of the parallel mechanism $\boldsymbol{K}_{\mathrm{p}}$ and $\boldsymbol{K}_{\mathrm{a}}$ at each position and pose of orthogonal experiment can be acquired by stiffness model and finite element analysis, respectively. And then correction coefficient matrix $C_{\mathrm{p}}$ at these position and poses can be acquired by dividing each element of matrix $K_{\mathrm{a}}$ by the corresponding element of matrix $\boldsymbol{K}_{\mathrm{p}}$, which can be used as the raw data of correction coefficient to be fitted. The relation between correct coefficients $c_{\mathrm{p} i i}$ and three independent end variables are discussed through the qualitative analysis of experimental results firstly, and then the mathematical expression of each correction coefficient $c_{\text {pii }}$ with respect to three independent end variables is acquired by using the least squares method to fit the raw data of correction coefficient.

Correction coefficient $c_{\mathrm{p} 11}$ is taken as an example to analyze its functional relation with three independent end variables. The data of orthogonal experiment can be divided into 7 groups by the values of variable $\theta$ from $-15^{\circ}$ to $15^{\circ}$. Figure 5(a) shows the mean of correction coefficient $c_{\mathrm{p} 11}$ of each group, which indicates an approximate quadratic function relation between correction coefficient $c_{\mathrm{p} 11}$ and variable $\theta$. Similarly, a quadratic function relation between correction coefficient $c_{\mathrm{p} 11}$ and variable $\sigma$ and a linear function relation between correction coefficient $c_{\mathrm{p} 11}$ and variable $Z_{0}$ are found through analyzing Figure 5(b) and (c).

Similarly, through the analysis on the rest main diagonal elements of correction coefficient matrix, it can be found that all the diagonal elements $c_{\mathrm{p} i i}$ have the quadratic function relation with variables $\theta$ and $\sigma$, and the linear relation with variable $Z_{0}$. Therefore, the vector of correction coefficients $c_{\mathrm{p} i i}$ can be expressed by Eq. (11):

$$
\left\{\begin{array}{l}
{\left[c_{\mathrm{p} 11}, c_{\mathrm{p} 22}, c_{\mathrm{p} 33}, c_{\mathrm{p} 44}, c_{\mathrm{p} 55}, c_{\mathrm{p} 66}\right]^{\prime}=\left[\boldsymbol{e}_{1}, \boldsymbol{e}_{2}, \boldsymbol{e}_{3}, \boldsymbol{e}_{4}, \boldsymbol{e}_{5}, \boldsymbol{e}_{6}\right]^{\prime} \cdot \times \boldsymbol{e} \boldsymbol{p}^{\prime}} \\
\boldsymbol{e}_{i}=\left[e_{i 1}, e_{i 2}, e_{i 3}, e_{i 4}, e_{i 5}, e_{i 6}, e_{i 7}, e_{i 8}, e_{i 9}\right], i=1, \ldots, 6, \\
\boldsymbol{e p}=\left[\theta^{2}, \theta, \sigma^{2}, \sigma, Z_{0}, \theta \times \sigma, \theta \times Z_{0}, \sigma \times Z_{0}, 1\right]
\end{array}\right.
$$

In each group of orthogonal experiment, one variable keeps unchanged while the other two variables are assigned seven different combinations of values. When the combination of values of the rest two variables has an obvious effect on the relation between the correction coefficient and the first variable, the correction coefficient cannot change with the first variable in a certain regular pattern. Therefore, the combined items of any two variables in Eq. (11), which have less influence on the

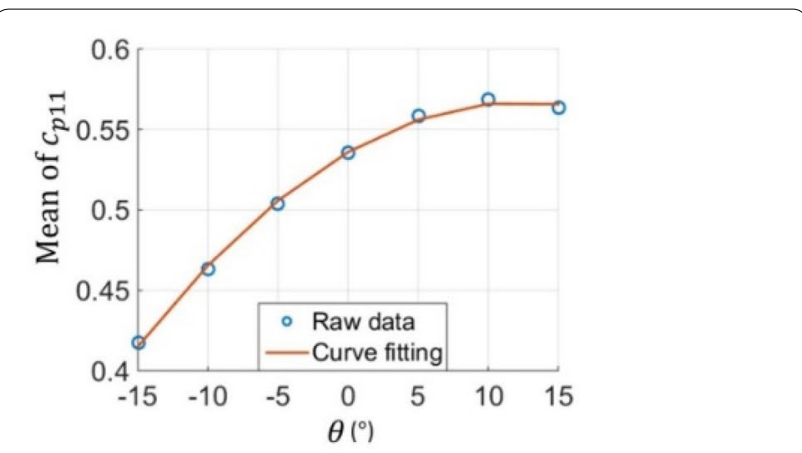

a Mean of $c_{\mathrm{p} 11}$ changing over end variable $\theta$

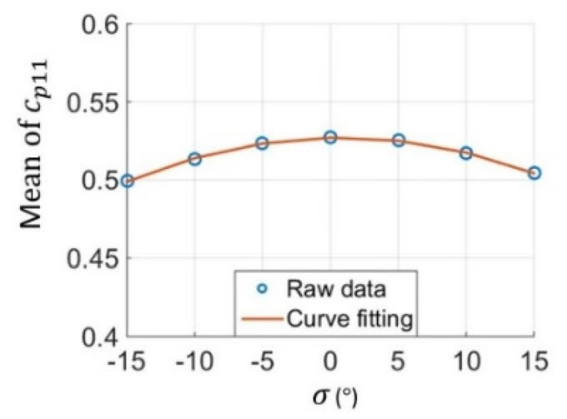

b Mean of $c_{\mathrm{p} 11}$ changing over end variable $\sigma$

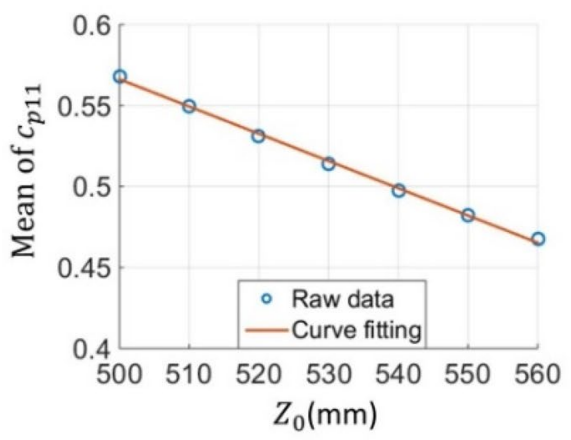

c Mean of $c_{\mathrm{p} 11}$ changing over end variable $Z_{0}$

Figure 5 The tendency of correction coefficient $c_{p 11}$

correction coefficient, can be ignored to simplify the fitting function.

The relation between correction coefficient $c_{\mathrm{p} 11}$ and three independent end variables $\theta, \sigma, Z_{0}$ are shown in Figure 6 . The data with the same marker in each subFigure come from the experiments with the same value of the corresponding variable. It can be seen from Figure 6(a) and (c) that correction coefficient $c_{\mathrm{p} 11}$ changes regularly with variables $\theta$ and $Z_{0}$. But the relation between correction coefficient $c_{\mathrm{p} 11}$ and variable $\sigma$ shown in Figure 6(b) 


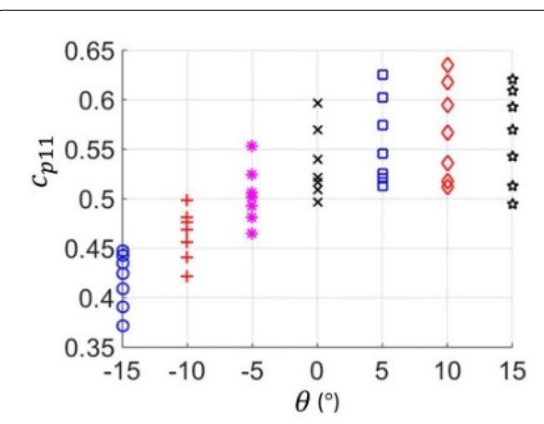

a Relation of $c_{\mathrm{p} 11}$ with the combination of variables $\sigma$ and

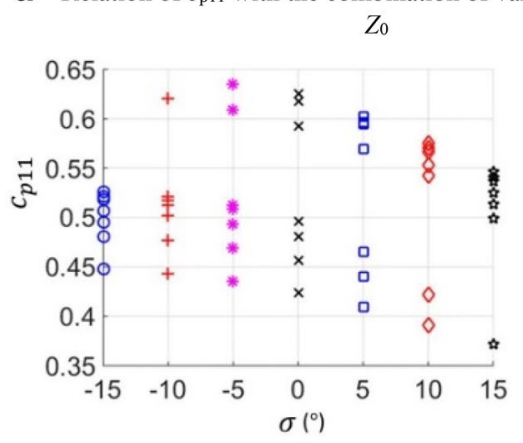

b Relation of $c_{\mathrm{p} 11}$ with the combination of variables $\theta$ and $Z_{0}$

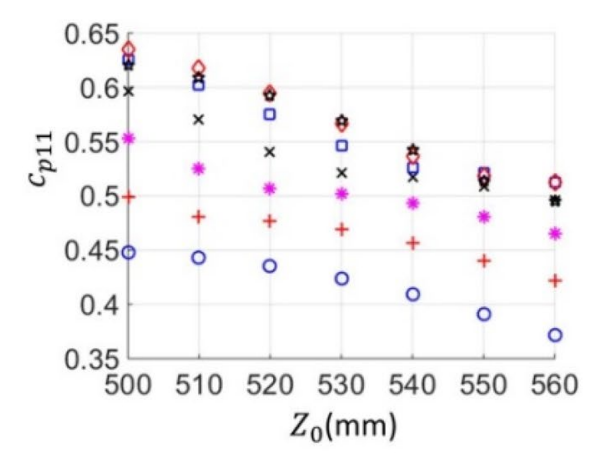

C Relation of $c_{\mathrm{p} 11}$ with the combination of variables $\theta$ and $\frac{\sigma}{\circ \theta=-15^{\circ}+\theta=-10^{\circ}, \theta=-5^{\circ} \times \theta=0^{\circ} \circ \theta=5^{\circ} \circ \theta=10^{\circ} \otimes \theta=15}$

Figure 6 Relation of $c_{p 11}$ with the combination of any two variables of $\theta, \sigma, Z_{0}$

is irregular. Therefore, the combined items of variables $\theta$ and $\sigma$, variables $\sigma$ and $Z_{0}$ play a very small role in the fitting function, which can be negligible by setting their coefficient to zero. The combined items of variables $\theta$ and $Z_{0}$ has such a marked impact on correction coefficient $c_{\mathrm{p} 11}$ that it cannot be ignored in Eq. (11).

Similarly, through the analysis on the other main diagonal elements of the correction coefficient matrix, it can be found that the influence of combined items of variables $\theta$ and $\sigma$ and variables $\theta$ and $Z_{0}$ are much smaller than that of variables $\sigma$ and $Z_{0}$ on correction coefficients $c_{\mathrm{p} 22}$, $c_{\mathrm{p} 33}$ and $c_{\mathrm{p} 66}$. And the combined item of variables $\theta$ and $Z_{0}$ plays a more important role on the fitting function of correction coefficient $c_{\mathrm{p} 44}$ than that of variables $\theta$ and $\sigma$, and variables $\sigma$ and $Z_{0}$. The combined items of any two variables all have little impact on correction coefficient $c_{\mathrm{p} 55}$.

Therefore, the fitting functions of correction coefficients $c_{\text {pii }}(i=1, \cdots, 6)$ about three independent end variables can be expressed as Eq. (12):

$$
\left[\begin{array}{c}
c_{\mathrm{p} 11} \\
c_{\mathrm{p} 22} \\
c_{\mathrm{p} 33} \\
c_{\mathrm{p} 44} \\
c_{\mathrm{p} 55} \\
c_{\mathrm{p} 66}
\end{array}\right]=\left[\begin{array}{ccccccccc}
e_{11} & e_{12} & e_{13} & e_{14} & e_{15} & 0 & e_{17} & 0 & e_{19} \\
e_{21} & e_{22} & e_{23} & e_{24} & e_{25} & 0 & 0 & e_{28} & e_{29} \\
e_{31} & e_{32} & e_{33} & e_{34} & e_{35} & 0 & 0 & e_{38} & e_{39} \\
e_{41} & e_{42} & e_{43} & e_{44} & e_{45} & 0 & e_{47} & 0 & e_{49} \\
e_{51} & e_{52} & e_{53} & e_{54} & e_{55} & 0 & 0 & 0 & e_{59} \\
e_{61} & e_{62} & e_{63} & e_{64} & e_{65} & 0 & 0 & e_{68} & e_{69}
\end{array}\right] \times\left[\begin{array}{c}
\theta^{2} \\
\theta \\
\sigma^{2} \\
\sigma \\
Z_{0} \\
\theta \times \sigma \\
\theta \times Z_{0} \\
\sigma \times Z_{0} \\
1
\end{array}\right] .
$$

The coefficients $e_{i j}(i=1, \ldots, 6, j=1, \ldots, 9)$ in the above equation can be evaluated by least square fitting based on 49 sets data of orthogonal simulation experiments. Thus, the quantitative functional relations between six main diagonal elements of correction coefficient matrix and three end variables are obtained, which are used to correct the linear stiffnesses and torsional stiffnesses along $x, y$ and $z$ axes in coordinate system $A-u v w$ of the end of the parallel mechanism at different position and poses.

\section{Verification and Analysis on Stiffness of the Parallel Mechanism}

As an important performance of PKM, stiffness should be fixed attention on especially in mechanical machining. In order to further apply the improved stiffness model, it is necessary to verify the accuracy and also study the stiffness distribution of parallel mechanism in the workspace.

\subsection{Verification of Improved Stiffness Model}

The verification of improved stiffness model includes two parts: the effectiveness of fitting correction coefficient in improving the accuracy of basic stiffness model; the accuracy of improved stiffness model by comparing with the stiffness obtained by ANSYS finite element simulation.

To evaluate the effect of correction coefficient matrix on the improvement of basic stiffness model of the parallel mechanism, verification experiment is conducted at more position and poses to achieve richer experiment data for regression analysis. Besides the 49 position and 
poses of orthogonal experiment, the additional 25 position and poses of the parallel mechanism which cover its workspace as widely as possible are selected to calculate the end stiffness matrices $\boldsymbol{K}_{\mathrm{p}}, \boldsymbol{K}_{\mathrm{m}}$ and $\boldsymbol{K}_{\mathrm{a}}$ by the basic stiffness model, the improved stiffness model and finite element analysis, respectively.

Correction effect matrix $\boldsymbol{M E}$, defined as Eq. (13), is introduced to evaluate the correction effect of correction coefficients $c_{\mathrm{p} i i}(i=1, \ldots, 6)$ quantitatively. If the values of $m e_{i i}$ are positive, it indicates that the stiffness from improved stiffness model is closer to the stiffness obtained by ANSYS finite simulation than the one from basic stiffness model. The larger the value of $m e_{i i}$ is, the better the correction effect of correction coefficient.

$$
\left\{\begin{array}{l}
\boldsymbol{M E}=\operatorname{diag}\left[m e_{11}, m e_{22}, m e_{33}, m e_{44}, m e_{55}, m e_{66}\right], \\
m e_{i i}=1-\left(k_{i i}^{m}-k_{i i}^{a}\right) /\left(k_{i i}^{p}-k_{i i}^{a}\right), i=1, \ldots, 6,
\end{array}\right.
$$

where $k_{i i}^{p}, k_{i i}^{m}, k_{i i}^{a}$ are the main diagonal elements of stiffness matrices obtained by basic stiffness model, improved stiffness model and finite element analysis, respectively.

As listed in Table 3, if correction effect $m e_{i i}$ ranges from 0.9 to 1 , the correction effect of correction coefficients $c_{\mathrm{p} i i}$ is satisfactory; if correction effect $m e_{i i}$ ranges from 0.6 to 0.9 , the correction effect is acceptable; if correction effect $m e_{i i}$ ranges from 0 to 0.6 , the correction effect is poor.

According to Table 3, the minimum of correction effect $m e_{i i}$ is equal to 0.32 , which proves that the modification method can effectively decrease the differnce between the stiffness obtained by basic stiffness model and finite element analysis. By comparing the mean and distribution of correction effect $m e_{i i}$ in orthogonal experiment and verification experiment, it can be seen that the correction effect in verification experiment is not as good as that in orthogonal experiment. It is due to the relatively small number of position and poses selected in orthogonal experiment to characterize the entire workspace of the parallel mechanism resulting in the relatively poor fitting precision of correction coefficient at the position and poses in verification experiment. Therefore, the correction effect can be obviously enhanced by appropriately increasing the number of level in orthogonal experiment to obtain the high-precision fitting correction coefficient.

In order to verify the accuracy of improved stiffness model, the relative errors of main diagonal elements of improved stiffness model $r e_{i i}(i=1, \ldots, 6)$ are defined as Eq. (14). The closer to zero the values of $r e_{i i}$ is, the more accurate the improved stiffness model.

$$
r e_{i i}=\frac{\left|k_{i i}^{m}-k_{i i}^{a}\right|}{k_{i i}^{a}} \times 100 \%, i=1, \ldots, 6 .
$$

The values of $r e_{i i}$ can be solved through the analysis on experimental data obtained by orthogonal experiment and verification experiment, which are shown in Table 4. The means of relative errors are all smaller than

Table 4 Statistics on relative errors $r e_{i i}$ of main diagonal elements of stiffness matrix

\begin{tabular}{lllrl}
\hline & Mean & Stdev & Max & Min \\
\hline$r e_{11}$ & 2.33 & 0.85 & 4.57 & 0.45 \\
$r e_{22}$ & 8.82 & 1.83 & 14.33 & 0.82 \\
$r e_{33}$ & 5.67 & 0.81 & 12.99 & 0.20 \\
$r e_{44}$ & 1.11 & 0.05 & 2.59 & 0.02 \\
$r e_{55}$ & 2.35 & 0.04 & 4.32 & 0.19 \\
$r e_{66}$ & 1.10 & 0.04 & 1.62 & 0.06 \\
\hline
\end{tabular}

\begin{tabular}{|c|c|c|c|c|c|c|c|c|}
\hline & \multirow[t]{2}{*}{ Correction effect } & \multirow[t]{2}{*}{ Mean } & \multirow[t]{2}{*}{ Stdev } & \multirow[t]{2}{*}{ Max } & \multirow[t]{2}{*}{ Min } & \multicolumn{3}{|c|}{ Distribution } \\
\hline & & & & & & {$[0.9,1]$} & {$[0.6,0.9)$} & {$[0,0.6)$} \\
\hline \multirow[t]{6}{*}{ Orthogonal experiment } & $m e_{11}$ & 0.96 & 0.01 & 0.98 & 0.95 & 49 & 0 & 0 \\
\hline & $m e_{22}$ & 0.90 & 0.12 & 1.00 & 0.53 & 36 & 12 & 1 \\
\hline & $m e_{33}$ & 0.98 & 0.01 & 1.00 & 0.97 & 49 & 0 & 0 \\
\hline & $m e_{44}$ & 1.00 & 0.00 & 1.00 & 1.00 & 49 & 0 & 0 \\
\hline & $m e_{55}$ & 1.00 & 0.00 & 1.00 & 1.00 & 49 & 0 & 0 \\
\hline & $m e_{66}$ & 1.00 & 0.00 & 1.00 & 0.99 & 49 & 0 & 0 \\
\hline \multirow[t]{6}{*}{ Verification experiment } & $m e_{11}$ & 0.83 & 0.12 & 1.00 & 0.37 & 3 & 20 & 2 \\
\hline & $m e_{22}$ & 0.69 & 0.16 & 0.91 & 0.32 & 1 & 18 & 6 \\
\hline & $m e_{33}$ & 0.68 & 0.07 & 0.73 & 0.44 & 0 & 23 & 2 \\
\hline & $m e_{44}$ & 0.99 & 0.00 & 1.00 & 0.99 & 25 & 0 & 0 \\
\hline & $m e_{55}$ & 0.99 & 0.00 & 0.99 & 0.98 & 25 & 0 & 0 \\
\hline & $m e_{66}$ & 0.93 & 0.02 & 0.98 & 0.86 & 24 & 1 & 0 \\
\hline
\end{tabular}

Table 3 Correction effect $m e_{i i}$ of correction coefficient $c_{\text {pii }}$ on main diagonal elements of stiffness matrix 


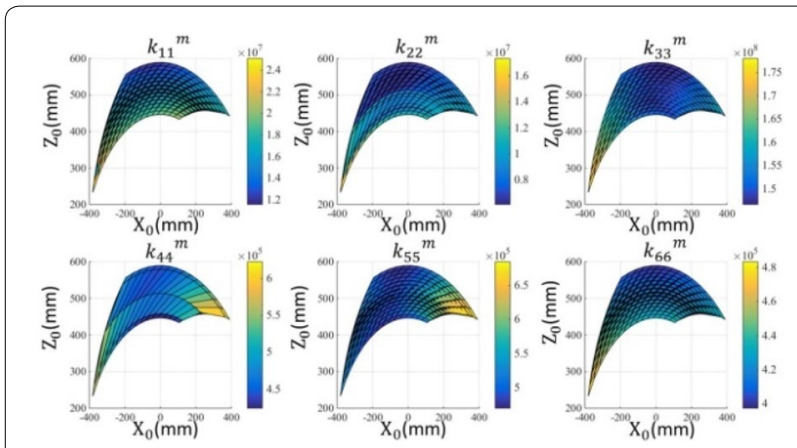

Figure 7 Distribution of stiffness over the workspace of the parallel mechanism

$10 \%$, which indicates that the improved stiffness model is accurate enough to describe the stiffness of the parallel mechanism in the mass. Through the analysis on standard deviation, maximum and minimum of $r e_{i i}$, the deviation of $r e_{22}$ is relatively large. It indicates that the improved stiffness model doesn't describe the liner stiffness along $y$ axis in coordinate system $A-u v w$ as effectively as others.

\subsection{Analysis on the Stiffness of the Parallel Mechanism}

To visualize the stiffness of the parallel mechanism, the distribution of stiffness over its workspace is calculated based on the improved stiffness model and then plotted.

It can be seen from the kinematics analysis that the workspace of the parallel mechanism is a plane $Y_{0}=0$, which is $x z$ plane of coordinate system $B$-xyz. The distribution of the main diagonal elements of stiffness matrix over the workspace is shown in Figure 7.

By comparing the distribution of stiffness $k_{11}^{m}, k_{22}^{m}$ and $k_{33}^{m}$, it can be seen that the linear stiffness of the parallel mechanism is relatively large in area $X_{0} \in[-400$, - 200], which is located at the extreme position and pose of workspace. When $X_{0}$ keeps fixed, the linear stiffness of the parallel mechanism decreases with the increment of $Z_{0}$. The value of $k_{33}^{m}$ is about one order of magnitude higher than that of $k_{22}^{m}$ and $k_{33}^{m}$, which indicates that the linear stiffness along $z$ axis of coordinate system $A-u v w$ of the parallel mechanism is the highest and the linear stiffnesses along $x$ and $y$ axes are similar in value.

By comparing the distribution of stiffness $k_{44}^{m}, k_{55}^{m}$ and $k_{66}^{m}$, it can be seen that the values of $k_{44}^{m}$ and $k_{55}^{m}$ are large in area $X_{0} \in[200,400]$ and the value of $k_{66}^{m}$ is large in area $X_{0} \in[-400,-200]$, which are all located at the extreme position and poses of workspace. As shown in Figure 7, the linear stiffnesses of the parallel mechanism is much better than its rotational stiffnesses so that the parallel mechanism can sustain greater forces in the directions of $x, y$ and $z$ axes of coordinate system $A-u v w$ rather than moments around $x, y$ and $z$ axes.

In the application of PKM in machining, the working stiffness in which the stiffness of parallel mechanism plays a key role is usually taken into account for the good machining quality. Therefore, according to the characteristics of stiffness distribution, the working stiffness of PKM in its application can be improved by optimizing its working position and pose. For example, in the robotic machining, the flange or fixture used to install the motorized spindle into the end of robot can be designed to ensure that the main force is applied along $\mathrm{z}$ axis of parallel mechanism, which can reduce the influence of deformation on the machining precision [25]. Moreover, the external axis also can help to optimize the machining position and pose of PKM for higher working stiffness. The following section presents the application of improved stiffness model to optimize the relative position of the workpiece and the PKM via external axis for the improvement of working stiffness of the PKM.

\section{Application of Improved Stiffness Model}

In order to expand the workspace of PKM for the machining of large-scale workpieces, the additional machinery are usually integrated into the machining system as the external axes of PKM [26-31]. However, there are few researches on the control and planning of movement of additional machinery which is generally determined by manual work according to the on-site teaching. Therefore, based on the improved stiffness model and the kinematics model, a way to find the most appropriate position of the additional machinery which makes the best working stiffness of the PKM is proposed.

\subsection{Optimization Algorithm on Position of Additional Machinery}

The external axes of PKM can be the linear axis along $x, y$ or $z$ axis of world coordinate system, the rotation axis around $x, y$ or $z$ axis of world coordinate system or any combination of them. However, too many external axes will increase the cost and difficulties in control of machining application. Therefore, the common additional machinery used to expand the workspace of PKM include: the cross slide table [26-28] providing two external linear axes; both the positioner $[29,30]$ and the slewing platform [31] providing an external rotation axis.

Taking the cross slide as an example, the most appropriate position of the additional machinery which makes the best working stiffness of the PKM can be determined by the following steps. 
(1) Establish four coordinate systems.

The coordinate system $B-x y z$ is established on the fixed platform of the parallel mechanism; the world coordinate system $W-x y z$ is established on the ground as intermediate transformation coordinate system; the cross slide table coordinate system $C$-xyz is established for controlling the motion of cross slide table; the worktable coordinate system $U-x y z$ is established for describing the trajectory to be machined.

(2) Determine the object to be optimized.

According to the form of external axes, the object to be optimized is defined as $d x$ and $d y$, which are the $x$ and $y$ coordinate values of the origin of worktable coordinate system $U$-xyz in world coordinate system $W$-xyz. According to the travel of cross slide table, $d x$ and $d y$ should be chosen in the allowable range $\left[d x_{\min }, d x_{\max }\right]$ and $\left[d y_{\min }, d y_{\max }\right]$.

(3) Get the position and pose matrices of machining trajectory in coordinate system $B-x y z$.

The position and pose matrices of machining trajectory are usually given in coordinate system $U$-xyz as $T_{i}^{U-x y z}$ according to the three-dimensional model of workpiece, which should be transformed into coordinate system $B$ - xyz through Eq. (15):

$\boldsymbol{T}_{i}^{B-x y z}(d x, d y)=\left(\boldsymbol{T}_{B}^{W}\right)^{-1} \times \boldsymbol{T}_{U}^{W}(d x, d y) \times \boldsymbol{T}_{i}^{U-x y z}$,

where $i$ is the number of machining position and poses; $\boldsymbol{T}_{B}^{W}$ is the transformation matrix of coordinate system $B-x y z$ relative to the world coordinate system $W-x y z ; \boldsymbol{T}_{U}^{W}(d x, d y)$ is the transformation matrix of the worktable coordinate system $U$-xyz relative to the world coordinate system $W-x y z$.

(4) Establish the optimization model.

Based on the position and pose matrices $\boldsymbol{T}_{i}^{B-x y z}(d x$, $d y$ ) obtained in step (3), the stiffness matrices of the parallel mechanism at each position and pose can be obtained by the improved stiffness model obtained in Section 4.2 and then the objective function can be constructed as Eq. (16):

$$
\operatorname{Min}(C(d x, d y))=\sum_{i=1}^{n} \frac{1}{K_{p i}\left(\boldsymbol{T}_{i}^{B-x y z}(d x, d y)\right)},
$$

where $K_{p i}\left(\boldsymbol{T}_{i}^{B-x y z}(d x, d y)\right)$ is the sum of the principle stiffness values of the parallel mechanism of machining position and pose $i$.

(5) Search the optimal solution.
(5.1) Randomly generate a population including multiple candidate solutions $(d x, d y)$ within their corresponding allowance range.

(5.2) Calculate the position and pose matrices $\boldsymbol{T}_{i}^{B-x y z}(d x, d y)$ by Eq. (14).

(5.3) Determine whether solution $(d x, d y)$ is the feasible solution by whether position and pose matrices $\boldsymbol{T}_{i}^{B-x y z}(d x, d y)$ are all in the workspace of the PKM.

(5.4) Calculate the value of objective function by Eq. (15) for each candidate solution $(d x, d y)$. Keep the minimum value of objective function as the current optimal value and the corresponding solution $(d x, d y)$ as the current optimal solution.

(5.5) Determine whether the number of iterations reaches the set value. If the set value is not reached, generate the next generation of group consisting of multiple candidate solutions $(d x, d y)$ by crossover and mutation operation, and then return to step (5.2). If the set value is reached, end the optimization and then output the optimal solution $(d x, d y)$.

To facilitate the motion control of cross slide table, it is necessary to convert the optimal position $(d x, d y)$ of worktable in world coordinate system $W$-xyz into that in cross slide table coordinate system $C-x y z$ by coordinate transformation.

\subsection{Case Study}

To verify the optimization method mentioned above, the plate workpiece shown in Figure 8 is taken as an example where there are two series of holes distributed in areas A and $\mathrm{B}$ respectively. The cross slide table drives the workpiece to let every series of holes to be machined enter the workspace of the PKM one after another. Thus, the movement of cross slide table is optimized to determine the relative position of the workpiece and the PKM which always makes the best working stiffness.

In the optimization, the values of objective function $C(d x, d y)$ for areas A and B keep decreasing, which

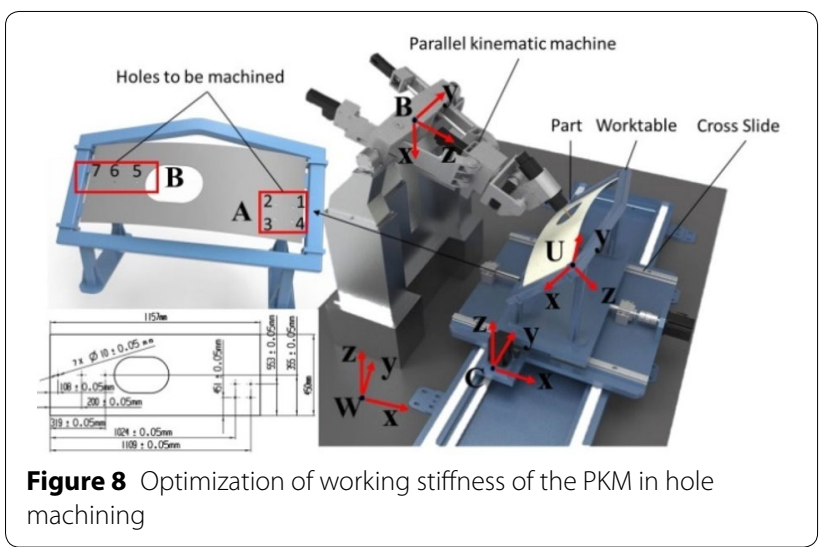


Table 5 Stiffness and position variables of the parallel mechanism for drilling each hole when the worktable locates at the optimal position

\begin{tabular}{|c|c|c|c|c|c|c|c|c|}
\hline No. of holes & $\begin{array}{l}k_{11}^{m} \\
\left(10^{7} \mathrm{~N} / \mathrm{m}\right)\end{array}$ & $\begin{array}{l}k_{22}^{m} \\
\left(10^{7} \mathrm{~N} / \mathrm{m}\right)\end{array}$ & $\begin{array}{l}k_{33}^{m} \\
\left(10^{8} \mathrm{~N} / \mathrm{m}\right)\end{array}$ & $\begin{array}{l}k_{44}^{m} \\
\left(10^{5} \mathrm{~N} \cdot \mathrm{m} / \mathrm{rad}\right)\end{array}$ & $\begin{array}{l}k_{55}^{m} \\
\left(10^{5} \mathrm{~N} \cdot \mathrm{m} / \mathrm{rad}\right)\end{array}$ & $\begin{array}{l}k_{66}^{m} \\
\left(10^{5} \mathrm{~N} \cdot \mathrm{m} / \mathrm{rad}\right)\end{array}$ & $X_{0}(\mathrm{~mm})$ & $Z_{0}(\mathrm{~mm})$ \\
\hline 1 & 2.02 & 1.32 & 1.67 & 5.36 & 5.31 & 4.52 & -307.38 & 363.45 \\
\hline 2 & 2.02 & 1.30 & 1.64 & 4.77 & 4.85 & 4.48 & -232.79 & 375.90 \\
\hline 3 & 2.24 & 1.51 & 1.70 & 4.83 & 4.66 & 4.63 & -263.39 & 315.72 \\
\hline 4 & 2.26 & 1.52 & 1.73 & 5.55 & 5.25 & 4.67 & -338.00 & 300.14 \\
\hline 5 & 1.95 & 1.23 & 1.62 & 4.61 & 5.12 & 4.45 & -156.24 & 433.25 \\
\hline 6 & 2.03 & 1.29 & 1.65 & 4.69 & 5.43 & 4.53 & -184.17 & 447.83 \\
\hline 7 & 2.20 & 1.38 & 1.71 & 4.83 & 5.42 & 4.62 & -206.45 & 458.46 \\
\hline
\end{tabular}

indicates the growing working stiffness for machining two series of holes. The improvement of working stiffness is described by the ratio $Q$ of the difference between the optimized stiffness and the maximum stiffness to the difference between the lowest stiffness recorded during the optimization and the maximum stiffness. If the value of ratio $Q$ is less than 1 , the working stiffness is improved. The smaller the value of ratio $Q$, the better the optimization efficiency. The average value of ratio $Q$ of six stiffnesses of machining trajectory in area $\mathrm{A}$ is 0.73 and that in area $\mathrm{B}$ is 0.74 , which indicates that the optimized working stiffness comes much closer to the maximum stiffness. The best positions $(d x, d y)$ of the worktable for machining two series of holes in areas A and B are $(-176,300)$ and $(-152,-136)$, respectively. The great difference between two best positions demonstrates the significance of the proposed optimization method in enhancing the working stiffness of the PKM.

By comparing the data listed in Table 5 with the distribution of stiffness over the workspace of the parallel mechanism shown in Figure 7, it can be seen that all the values of stiffness $k_{11}^{m}, k_{22}^{m}, k_{33}^{m}, k_{44}^{m}$ and $k_{66}^{m}$ after optimizing the position of worktable concentrate near their maximal values except the values of stiffness $k_{55}^{m}$.

When the worktable locates at position $(-176,300)$ in $W$ - xyz, the PKM drills holes 1 to 4 with the position variable $X_{0}$ of the parallel mechanism ranging from -400 to -200 , where other than stiffness $k_{55}^{m}$ almost any other stiffness is relatively high. It conforms with the finding discussed in Section 5.2. When the worktable locates at position $(-152,-136)$ in $W-x y z$, the PKM drills holes 5 to 7 with the position variable $X_{0}$ of the parallel mechanism ranging from -210 to -150 , where the stiffness is a little poorer. It is because the disturbed holes 5 to 7 cannot be drilled together by the PKM with the position variable $X_{0}$ of the parallel mechanism ranging from -400 to -200 . Therefore, a series of suboptimal positions of the parallel mechanism is found, which ensures the best working stiffness of the PKM when the series of holes in area $\mathrm{B}$ is machined without moving the worktable.
This case proves that the proposed optimization method can solve the best position of worktable to obtain the highest working stiffness of the parallel mechanism and also ensure the machining trajectory in the workspace of the PKM.

\section{Conclusions}

The studies on kinematics modeling and stiffness modeling of a novel 5-DOF PKM with redundant constraints are conducted, which promote the theoretical research method of the PKM and improve its application in the field of machining.

(1) To facilitate the study on kinematics, the parallel mechanism of the PKM is transformed into a joint of the PKM, which is equivalent to a 3-joint series mechanism. The kinematics analysis is firstly carried out on the equivalent series mechanism of the PKM and then on the parallel mechanism.

(2) Correction coefficient matrix is introduced to improve the basic stiffness model of the parallel mechanism. Based on the data from orthogonal simulation experiment, the qualitative relation between correction coefficients and three independent end variables of the parallel mechanism is described by polynomial fitting function. The results of verification experiment prove the effectiveness of the modification method on improving the precision of main diagonal elements of stiffness matrix over the workspace of the parallel mechanism.

(3) Through the research on the application of kinematic model and improved stiffness model of the parallel mechanism in the field of machining, the working stiffness of the PKM is optimized by searching the best relative position of the PKM and workpiece to be machined for improving the machining accuracy. 


\section{Acknowledgements}

The authors wish to acknowledge Shanghai Machine Tool Works Co., Ltd, for the help in checking CAD drawings and designing manufacturing process of the proposed PKM.

\section{Authors' Contributions}

NS, JL conceived the idea of the study; LG, FY and ZY refined the ideas; LG performed the theoretical derivation and simulation experiment. FY contributed to structure design. LG and ZY and wrote the manuscript; ZW assisted with data analyses. All authors read and approved the final manuscript.

\section{Authors' Information}

Nanyan Shen is currently an associate professor/Ph.D at Shanghai Key Laboratory of Intelligent Manufacturing and Robotics, School of Mechatronic Engineering and Automation, Shanghai University, China. Her research interests include Intelligent Manufacturing and Robotics.

Liang Geng is currently a master candidate at School of Mechatronic Engineering and Automation, Shanghai University, China.

Jing Li is currently an associate professor/Ph.D at Shanghai Key Laboratory of Intelligent Manufacturing and Robotics, School of Mechatronic Engineering and Automation, Shanghai University, China. Her research interests include intelligent manufacturing and robotics.

Fei Ye is currently a master candidate at School of Mechatronic Engineering and Automation, Shanghai University, China.

Zhuang Yu is currently a master candidate at School of Mechatronic Engineering and Automation, Shanghai University, China.

Zirui Wang is currently a master candidate at School of Mechatronic Engineering and Automation, Shanghai University, China.

\section{Funding}

Not applicable.

\section{Competing Interests}

The authors declare no competing financial interests.

\section{Author Details}

1 Shanghai Key Laboratory of Intelligent Manufacturing and Robotics, Shanghai University, Shanghai 200444, China. ${ }^{2}$ School of Mechatronic Engineering and Automation, Shanghai University, Shanghai, China.

Received: 5 June 2019 Revised: 8 January 2020 Accepted: 16 April 2020 Published online: 13 May 2020

\section{References}

[1] TTang, J Zhang, M Ceccarelli. Static performance analysis of an exechonlike parallel kinematic machine. International Conference on Mechanism and Machine Science, Guangzhou, China, December 15-17, 2016: 831-843.

[2] J Zhang, Y G Li, T Huang. Dynamic modeling and eigenvalue evaluation of a 3-DOF PKM module. Chinese Journal of Mechanical Engineering, 2010, 23(2): 166-173.

[3] TF Tang, J Zhang. Conceptual design and kinetostatic analysis of a modular parallel kinematic machine-based hybrid machine tool for large aeronautic components. Robotics and Computer-Integrated Manufacturing, 2019, 57: 1-16.

[4] THuang, XY Zhao, D J Whitehouse. Stiffness estimation of a tripod-based PKM. Transactions on Robotics and Automation, 2002, 18(1): 50-58.

[5] Y Q Zhao, Y Jin, J Zhang. Kinetostatic modeling and analysis of an Exechon PKM(PKM) module. Chinese Journal of Mechanical Engineering, 2016, 29(1): 33-44.

[6] Z M Bi, Y Jin. Kinematic modeling of Exechon PKM robot. Robotics and Computer-Integrated Manufacturing, 2011, 27: 186-193.

[7] Z M Bi, Y Jin, R Gibson, et al. Kinematics of PKM Exechon. IEEE International Conference on Information and Automation, Zhuhai, Macau, China, June 22-24, 2009: 201-206.

[8] D Zhang, L Wang. Conceptual development of an enhanced tripod mechanism for machine tool. Robotics and Computer-Integrated Manufacturing, 2015, 21(4): 318-327.
[9] D Zhang, J Lei. Kinematic analysis of a novel 3-DOF actuation redundant parallel manipulator using artificial intelligence approach. Robotics and Computer-Integrated Manufacturing, 2011, 27: 157-163.

[10] K Nagai, Z Liu. A systematic approach to stiffness analysis of parallel mechanisms and its comparison with FEM. SICE Annual Conference, Takamatsu, Japan, September 17-20, 2007: 1543-1548.

[11] HT Liu, THuang, D G Chetwynd, et al. Stiffness modeling of parallel mechanisms at limb and joint/link. IEEE Transactions on Robotics, 2017 33(3): 734-741.

[12] C Dong, H Liu, W Yue, et al. Stiffness modeling and analysis of a novel 5-DOF hybrid robot. Mechanism and Machine Theory, 2018, 125(1): 80-93.

[13] D Deblaise, X Hernot, P Maurine. A systematic analytical method for PKM stiffness matrix calculation. International Conference on Robotics and Automation IEEE, Orlando, FL, USA, May 15-19, 2006: 4213-4219.

[14] Z M Bi. Kinetostatic modeling of Exechon PKM for stiffness analysis. The International Journal of Advanced Manufacturing Technology, 2014, 71(1-4): 325-335.

[15] C Dumas. Joint stiffness identification of six-revolute industrial serial robots. Robotics and Computer-Integrated Manufacturing, 2011, 27(4): 881-888.

[16] S Fan, SW Fan, W B Lan, et al. A new approach to enhance the stiffness of heavy-load parallel robots by means of the component selection. Robotics and Computer-Integrated Manufacturing, 2020, 161(1): 101834.

[17] FX He, Y Liu, K Liu. A chatter-free path optimization algorithm based on stiffness orientation method for robotic milling. The International Journal of Advanced Manufacturing Technology, 2018, 101(1): 2739-2750.

[18] J Li, B Li, NY Shen, et al. Effect of the cutter path and the workpiece clamping position on the stability of the robotic milling system. International Journal of Advanced Manufacturing Technology, 2017, 89(9-12): 1-15.

[19] H Bo. Kinematically identical manipulators for the Exechon parallel manipulator and their comparison study. Mechanism and Machine Theory, 2016, 103: 117-137.

[20] C Faria, F Ferreira, W Erlhagen, et al. Position-based kinematics for 7-DoF serial manipulators with global configuration control, joint limit and singularity avoidance. Mechanism and Machine Theory, 2018, 121(1): 317-334.

[21] J Zhang, Y Q Zhao, Y Jin. Kinetostatic-model-based stiffness analysis of Exechon PKM. Robotics and Computer-Integrated Manufacturing, 2016, 37(1): 208-220

[22] TF Tang, J Zhang. Conceptual design and comparative stiffness analysis of an Exechon-like parallel kinematic machine with lockable spherical joints. International Journal of Advanced Robotic Systems, 2017, 14(4): 1-13.

[23] G Yu, L Wang, JWu, et al. Stiffness modeling approach for a 3-DOF parallel manipulator with consideration of nonlinear joint stiffness. Mechanism and Machine Theory, 2018, 123(1): 137-152.

[24] A Raoofian, A Taghvaeipour, A Kamali. On the stiffness analysis of robotic manipulators and calculation of stiffness indices. Mechanism and Machine Theory, 2018, 130(1): 382-402.

[25] N Y Shen, Z M Guo, J Li, et al. A practical method of improving hole position accuracy in the robotic drilling process. The International Journal of Advanced Manufacturing Technology, 2018, 96(5-8): 2973-2987.

[26] F P Marcelo, L E Luis, P Hilde, et al. Analysis of a single-edge micro cutting process in a hybrid parallel-serial machine tool. International Journal of Mechanical Sciences, 2019, 151(1): 222-235.

[27] D Xie, J Zhu, M Wang, et al. Motion performance analysis and control mode design for the cross slide. IOP Conference Series Materials Science and Engineering, 2017, 244: 1-8.

[28] W J Tian, F W Yin, H T Liu, et al. Kinematic calibration of a 3-DOF spindle head using a double ball bar. Mechanism and Machine Theory, 2016, 102 $167-178$

[29] Y Liu, X Tian. Robot path planning with two-axis positioner for non-ideal sphere-pipe joint welding based on laser scanning. International Journal of Advanced Manufacturing Technology, 2019, 105(1-4): 1295-1310.

[30] T Bonnemains, H Chanal, B C Bouzgarrou, et al. Dynamic model of an overconstrained PKM with compliances: The Tripteor X7. Robotics and Computer-Integrated Manufacturing, 2013, 29(1): 180-191.

[31] S M Safavi, S S Mirian, R Abedinzadeh, et al. Use of PLC module to control a rotary table to cut spiral bevel gear with three-axis CNC milling. International Journal of Advanced Manufacturing Technology, 2010, 49(9-12): 1069-1077. 